\section{Die Universitäre Dermatologie in Kiel und die Dermatologenfamilie Schirren: Fruchtbare Wechselbeziehungen}

\author{
Dermatology in Kiel: Fruitful Correlation of the Departement of Dermatology \\ with the Family Schirren
}

\section{Zusammenfassung}

Die klinische und experimentelle Dermatologie entwickelte sich in Schleswig-Holstein und dessen Kapitale Kiel seit über 100 Jahren und ist gekennzeichnet durch ein enges Zusammenwirken der Universitären Dermatologie mit niedergelassenen Dermatologen. Hier ist die Dermatologenfamilie Schirren besonders hervorzuheben. Zwei Schwerpunkte in Forschung und Anwendung basieren auf dieser Kooperation: die Strahlenbiologie und Röntgentherapie sowie die Andrologie.
Abstract

Dermatology started in Kiel more than 100 years ago. There was a fruitful correlation between the clinical and experimental dermatology of the Department of Dermatology at the University of Kiel and the dermatologists in private praxis. Special emphasis is focused on the family Schirren. They are involved in two special topics of great interest: photobiology and radiotherapy on one hand and andrology on the other hand.
Die Medizingeschichte ist Teil unserer Kulturgeschichte. Sie kümmert sich meistens um Meilensteine der Medizin und deren Umfeld, sowie um die Wirkungsgeschichte von Personen. Diese können globale Wirkungen entfalten oder von Bedeutung sein für ihre Region. Solches gilt auch für die Geschichte unseres Faches [1], der Dermatologie und Venerologie, und hier im Besonderen für die Entwicklung über gute 100 Jahre in Holsteins Kapitale Kiel. Dies ist in engem Zusammenwirken mit der Dermatologischen Universitätsklinik Kiel (Tab. 1) und dem Kieler ärztlichen Verein geschehen. Die Dermatologenfamilie Schirren hat über 4 Generationen und weit gefächert unser Spezialfach dort mitentwickelt und vorangebracht [2]. Es geschah teils als niedergelassene Fachärzte und anderenteils als Hochschullehrer und Forscher im benachbarten Hamburg und entfernter in München und Marburg. In den drei Generationen III-V finden sich 15 Ärztinnen und Ärzte, darunter 9 Fachärzte für Dermatologie (Abb.1).

An zwei Schwerpunkten mit überregionaler und internationaler Ausstrahlung sind die Universität Kiel, die Dermatologie ebendort und die Familie Schirren in vielfältigen Facetten wesentlich an der Gestaltung und maßgeblich an der Durchführung betei- ligt. Einer dieser Schwerpunkte, derjenige zur Strahlenbiologie und Strahlentherapie, ging vor hundert Jahren von Kiel aus in alle Welt und durchwirkt unser Fach fortwährend, während der zweite, die Andrologie, von den Schirren's ausgeht und den Schwerpunkt in Hamburg nahm mit weltweiter Ausstrahlung.

\section{Zunächst die Familie Schirren}

Es begann mit Carl Schirren (1861 - 1921), der einer baltischen Familie aus Riga (Lettland) entstammend, sein Medizinstudium in Kiel und Freiburg i. Br. absolvierte und 1888 in Kiel mit dem Staatsexamen abschloss und promovierte. Als Assistent war er zuerst bei Prof. Friedrich von Esmarch an der Chirurgie in Kiel und anschließend beim Dermatologen Prof. Oskar Lassar in Berlin. Am 1. Oktober 1890 eröffnete er als erster niedergelassener Hautarzt in Schleswig-Holstein seine Praxis in der Landeshauptstadt Kiel am Schlossgarten 13. Schon 1888 wurde er in den Kieler Ärzteverein aufgenommen und seit 1890 war er aktives Mitglied der 1889 gegründeten Deutschen Dermatologischen Gesellschaft (DDG). 
Tab. 1 Die Direktoren der Universitätshautklinik Kiel

\begin{tabular}{|ll}
\hline $1902-1906$ & Ernst von Düring-Pascha, Extraordinarius \\
\hline $1906-1937$ & Victor Klingmüller, ab 1919 Ordinarius \\
\hline $1937-1943$ & Josef Vonkennel \\
\hline $1943-1950$ & Paul-Wilhelm Schmidt \\
\hline $1950-1975$ & Albin Proppe \\
\hline $1975-2005$ & Enno Christophers \\
\hline ab 2005 & Thomas Schwarz \\
\hline
\end{tabular}

Er vertrat unser Fach in der jeweils gestalteten Breite und der durch Forschung ausgeloteten Tiefe. Der Dermatopharmakologie war er in besonderer Weise zugewandt; er entwickelte zusammen mit Apothekern eine Vielzahl von angepassten Verfahren zur Lokaltherapie.

\section{Strahlenbiologie und Strahlentherapie, ein Kieler Schwerpunkt vor hundert Jahren}

Carl Schirren war einer der Pioniere der dermatologischen Röntgentherapie und schaffte sich in seiner Praxis schon 1904 ein Weichstrahlengerät an, womit er über Jahrzehnte Hauttumoren erfolgreich behandelte und neue Indikationen suchte. Dies führte auch zur engen Zusammenarbeit und stetem Austausch mit dem „Strahlenforschungsinstitut“, welches Hans Mayer (1877-1964) nach seiner Habilitation 1911 für „Röntgenkunde und Lichttherapie“ zunächst an der Dermatologie (Klingmüller), dann selbstständig in der Medizinischen Fakultät Kiel gründete [3]. Hans Mayer begann seine Assistentenzeit in Kiel bei von Düring, ging dann auf eine richtige „Kavalierstour“ durch Europa und durch die medizinischen Fächer, Dermatologie und Strahlenheilkunde verknüpfend. Er gründete 1912 die Zeitschrift Strahlentherapie und 1927 die „Deutsche Gesellschaft für Lichttherapie“. 1920 lehnte er den Ruf auf das Ordinariat der Derma- tologie in Greifswald ab und übernahm 1921 die Direktion der neu gegründeten „Röntgen- und Lichtabteilung“ seiner Heimatstadt Bremen. In Kiel existierte damals, vor dem ersten Weltkrieg, mit der Strahlenforschung ein interdisziplinärer, weltoffener Schwerpunkt der Forschung und der klinischen Anwendung mit großer Wirkung und internationaler Ausstrahlung. Daran war Carl Schirren, der niedergelassene Hautarzt, aktiv und wesentlich beteiligt. Damit aber nicht genug. Zwei Generationen später, nach dem zweiten Weltkrieg, greift sein Enkel Carl Georg Schirren (1923 - 1969) das Thema wieder auf und verhilft, neben der klinischen Dermatologie, vor allem der dermatologische Radiotherapie sowohl wissenschaftlich als auch in der klinischen Anwendung und Bestätigung (heute EBM) erneut zum Durchbruch. Er hat dies vordringlich an der Dermatologischen Universitätsklinik in München getan, und anlässlich eines Forschungsaufenthaltes bei Guido Miescher an der Dermatologischen Universitätsklinik in Zürich vertieft. Kurz nach der Übernahme des Dermatologischen Lehrstuhls an der Universität Marburg ist er, mitten im Aufbau, erkrankt und 1969 im Alter von 46 Jahren verstorben. Sein Werk blieb leider unvollendet. Seine Ideen aber und seine Therapieanweisungen wirken weiter und wurden vielfältig übernommen und fortentwickelt, nicht nur in Kiel.

\section{Die Universitäre Dermatologie in Kiel}

Im Jahre 1886, also nur 4 Jahre vor der Praxis Schirren, eröffnet Nicolaus Nicolai eine Privatklinik für Haut- und Geschlechtskrankheiten, so lesen wir bei Albrecht Scholz [4], und er übernahm auch die Vorlesung an der medizinischen Fakultät. Und erst 1902 wurde als erster Extraordinarius für Dermatologie Ernst von Düring-Pascha (1858-1944) berufen, der nur 4 Jahre blieb. Er kam von der Kaiserlichen Medizinschule Konstantinopel und ging schon 1906 weiter nach Dresden (Sanatorium Weißer Hirsch). In zwei angemieteten Privathäusern hielt er Sprechstunde und hatte 28 Betten. Eine eigene, neu zu bauende Klinik wurde ihm verwehrt. Ihm folgte Victor Klingmüller (1870 - 1942), der eigentliche Begründer der Universitären Der-

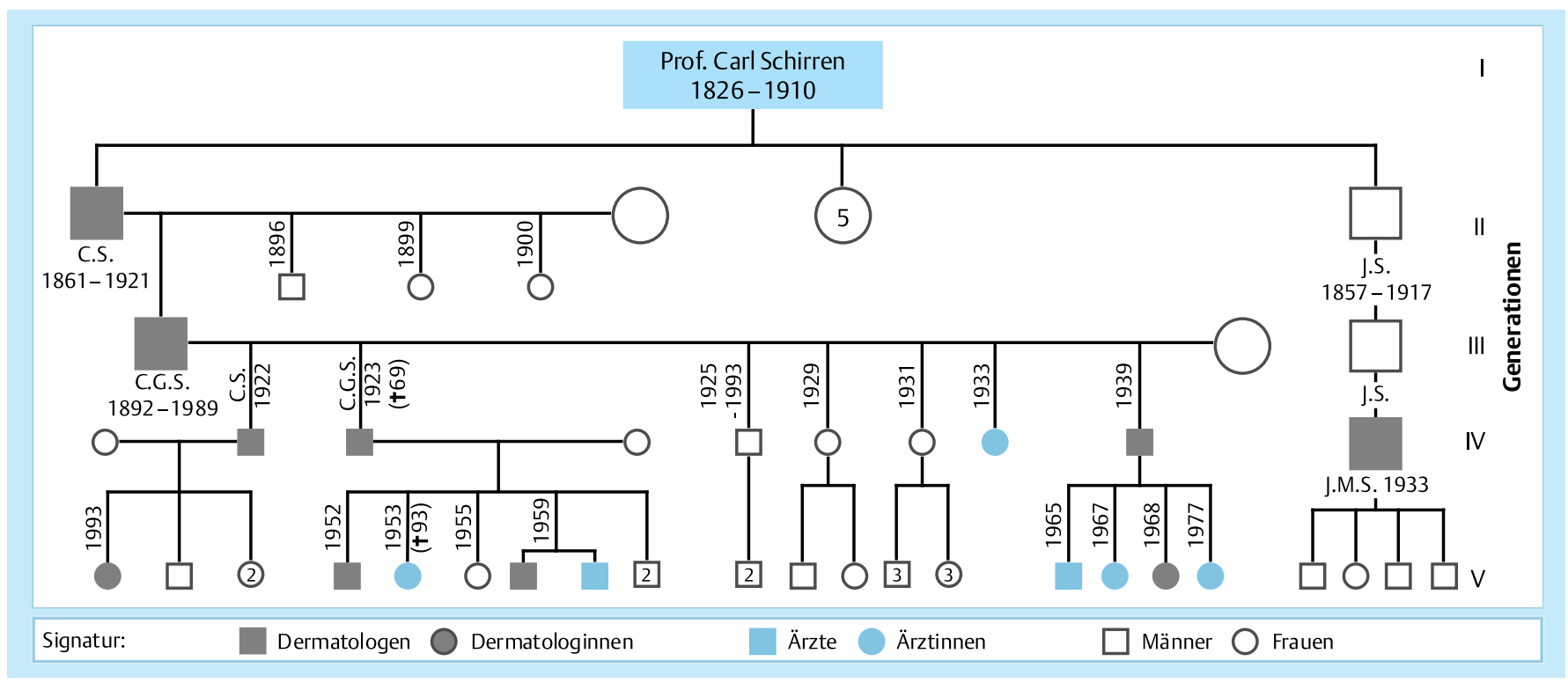

Abb. 1 Stammbaum der Familie Schirren. 
matologie in Kiel, die 1919 als Ordinariat eingerichtet wurde. Seither arbeiteten und forschten prominente Fachvertreter auf dem Kieler Lehrstuhl für Dermatologie und Venerologie mit großer Ausstrahlung im Raume Ostsee und Skandinavien, aber auch weltweit (Tab.1). Erst 1928 konnte die Dermatologie in Räumen der Medizinischen Klinik adäquat untergebracht werden und es dauerte bis ins Jahr 1968, als Albin Proppe eine neue und auf die modernen Bedürfnisse des Faches ausgerichtete Hautklinik an der Schittenhelmstraße 7 einweihen konnte. Diese blieb jahrzehntelang Vorbild und Modell für die Um- und Neubaupläne anderer Universitäten im In- und Ausland.

Carl Schirren führte seine Praxis bis kurz vor seinem Ableben und übergab diese im Herbst 1921 an seinen Sohn Carl Georg Schirren (1892-1989), der sein Medizinstudium in Kiel 1919 mit der Promotion abschloss und anschließend in Berlin (A. Isaac und M. Friedländer) zum Hautfacharzt ausgebildet wurde. Dies wurde notwendig, da er die Facharztweiterbildung in Kiel nicht absolvieren konnte. Der damalige Direktor, Prof. V. Klingmüller, befürchtete die Konkurrenz und lehnte ab, was zu einer hinhaltenden Spannung führte. Dennoch führte er die väterliche Praxis am Schlossgarten 13 weiter. Und dies trotz räumlicher Begehrlichkeiten der Kieler Handelskammer, welche das Gebäude im Auge hatte, weiter bis 1971, also 50 Jahre lang, und vertrat unser Fach in der gesamten Breite. Zudem entwickelte er erfolgreich besondere Schwerpunkte in der Berufsdermatologie und der Andrologie aus. Die Spannung mit der Kieler Universitätshautklinik wurde von Albin Proppe 1967 durch einen Besuch mit ehrenvoller Würdigung anlässlich des 75. Geburtstages ausgeräumt [5]. Zum Jahresbeginn 1972 übernahm sein jüngster Sohn Carl Hermann Schirren (1939) die Praxis. Er hatte in München und Kiel Medizin studiert, wo er 1965 promovierte. Die Assistentenzeit führte ihn über München und Koblenz nach Hamburg, wo er bis1971 an der Universitätshautklinik zum Hautfacharzt weitergebildet wurde. Im Jahre 1975 konnte er seinen Vetter Jul Michael Schirren (1933) als Partner gewinnen. Dieser studierte Medizin in Kiel, Berlin und wiederum in Kiel, wo er 1960 das Staatsexamen ablegte. Die Assistentenzeit führte ihn durch mehrere Kliniken, bis er an die Universitäts-Hautklinik Kiel kam, wo er 1967 die Weiterbildung zum Hautfacharzt abschloss. Er wurde Oberarzt und habilitierte sich für unser Fach 1974 ebendort.

Seit 1975 führten Carl Hermann und Jul Michael Schirren zusammen und erfolgreich eine dermatologische Gemeinschaftspraxis, immer noch da wo der Großvater begonnen hatte, am Schlosspark 13 in Kiel. Es wurde ausgeweitet und umgebaut, kräftig erneuert und spezialisiert, sodass die gewachsene Breite und die traditionelle Andrologie zusammen mit der verstärkten Allergologie Platz finden und sich weiter entwickeln konnten.
Andrologie, ein Schwerpunkt von Hamburg aus seit 50 Jahren

Carl Georg Schirren der Ältere führte in seiner Praxis die andrologische Diagnostik und Therapie schon vor gut 50 Jahren ein und sammelte reichlich Erfahrung, was sich zu einer besonderen Qualität seiner Praxis herausbildete. Dies wurde in der Gemeinschaftspraxis fortentwickelt durch seinen jüngsten Sohn Carl Hermann Schirren und seinen Neffen Jul Michael Schirren. Solches wäre nicht möglich gewesen ohne Vorbild und Führung. Dies verschafft ihnen, Vater, Sohn und Neffen, aber auch vielen Kollegen, der älteste Sohn, nämlich der 1922 geborene Carl Schirren der Jüngere, der jahrzehntelang als Universitäts-Professor an der Universitätshautklinik Hamburg-Eppendorf die Andrologie als ein klinischer Pionier und wissenschaftlicher Vorkämpfer gründete und weitete. Er hat ungezählte Kollegen in die Andrologie eingeweiht, fortgebildet und in ihrem selbstständigen Wirken begleitet. Er hat die Andrologie in unserem Fach stark gemacht, ihr Ansehen und Respekt verschafft, national und international. In kluger Voraussicht hat er auch frühzeitig die Weichen gestellt zur Bildung der fächerübergreifenden Reproduktionsmedizin, wie sie jetzt an den meisten Zentren betrieben wird. Dazu kommen Arbeitskreise, an denen auch die Gemeinschaftspraxis Schirren in Kiel tatkräftig und innovativ beteiligt ist. Carl Schirren hat sich auch der Familiengeschichte Schirren angenommen [2].

Die Praxis Schirren am Schlossgarten 13 in Kiel entwickelt sich ebenfalls weiter. Nachdem Jul Michael Schirren 1998 ausgeschieden ist, wurde sie 2005 ergänzt und neu gestaltet durch den $\mathrm{Zu}$ gewinn der Hautfachärzte Frau Martina Podszuweit und Herrn Dr. Stephan Lischner, die beide ihre Facharztweiterbildung an der Universitätshautklinik in Kiel absolvierten.

Mittlerweile gibt es in Kiel 17 Hautfacharztpraxen und die Universitäts-Hautklinik Kiel entwickelt mit dem neuen Direktor Prof. Dr. Thomas Schwarz (Tab.1) gerade ihr aktuelles und zukunftsgängiges Profil. Die Versorgung der hautkranken Patienten ist durch die gute und vielfältige Kooperation Praxis - Klinik bestens gewährleistet, die Lehre entwickelt moderne Inhalte und um die Forschung muss man sich nicht bange sein. Und wieder ist Photobiologie ein Schwerpunkt.

\section{Literatur}

${ }^{1}$ Jung EG. 100 Jahre Dermatologie in Deutschland. Akt Dermatol 2004; 30: $333-338$

${ }^{2}$ Schirren C. 100 Jahre Dermatologie in einer Familie. Kiel: Schmidt \& Klaunig, 1996

3 Jung EG. 75 Jahre Deutsche Gesellschaft für Lichtforschung. Akt Dermatol 2003; 29: 252-260

${ }^{4}$ Scholz A. Geschichte der Dermatologie in Deutschland. Heidelberg \& Berlin: Springer, 1999

${ }^{5}$ Proppe AS. Ein Leben für die Dermatologie. Berlin: Diesbach, 1993 